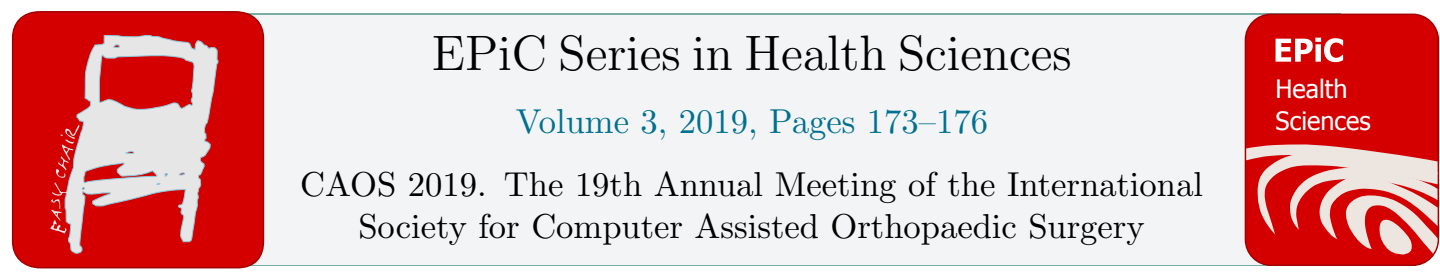

\title{
Depth Camera Augmented Fluoroscopy with Video Overlay
}

\author{
Matthew D Hickey ${ }^{1}$, Pierre Guy ${ }^{2}$, and Antony J Hodgson ${ }^{1,3}$ \\ Departments of ${ }^{1}$ Biomedical Engineering, ${ }^{2}$ Orthopedics $\&{ }^{3}$ Mechanical Engineering, \\ University of British Columbia, Vancouver, Canada \\ matthew.hickey@alumni.ubc.ca
}

\begin{abstract}
In many orthopedic surgeries, the surgeon relies on a C-arm fluoroscopy machine with the images usually displayed on a bedside monitor. The mental effort that surgeons expend transferring information from the imaging display back to the surgical site can lead to distraction causing errors that could directly influence quality of surgery. Depth Camera Augmented Fluoroscopy (DeCAF) uses an Intel RealSense depth camera to provide real-time visualization of the surgical site by overlaying $\mathrm{x}$-ray images from the C-arm onto live video of the patient's surface anatomy. Using geometric data acquired via the depth camera, the device facilitates transforming a real-time video feed aligned with the camera coordinate system to a perspective aligned with the x-ray source. The xray overlay is attained while restricting incursion on the surgeon's work area and allowing the $\mathrm{C}$-arm to be used in its normal position to minimize radiation exposure. DeCAF successfully facilitates an $\mathrm{x}$-ray video overlay feature while eliminating key limitations such as size, radiation exposure and acquisition time associated with other similar devices. Future work will involve evaluating overlay accuracy, the addition of second depth camera to aid in filling in areas with missing details, and a design iteration involving bagging of the camera with a sterile cover to ensure compliance with asepsis requirements prior to evaluating the system in the operating room.
\end{abstract}

\section{Introduction}

Many recent technological developments in the operating room (OR) seek to improve the efficacy of procedure planning and increase the precision of surgical tasks while reducing operative trauma [1]. In small incision surgeries, a surgeon's view of the subcutaneous anatomy of interest is at least partially obstructed by skin and soft tissue. Consequently, surgeons must rely on medical imaging systems to obtain this information, usually displayed on a bedside monitor. The mental effort that surgeons expend transferring information from the imaging display back to the surgical site can lead to distraction causing errors that could directly influence quality of surgery [2].

The CamC has been proposed by Navab et al., a device that augments a C-arm fluoroscope through the use of a video camera [3]. The technology provides a real-time visualization of the surgical site by 
overlaying x-ray images from the C-arm onto live video of the patient's surface anatomy. The technology was tested clinically by Heide et al. over 104 surgeries showing a $46 \%$ reduction in the number of x-ray shots, reducing the amount of patient and surgical staff radiation exposure with no significant difference in surgical time [4]. Despite the obvious advantages of this system, there are some major limitations. The CamC must be used in the inverted configuration, exposing the patient and surgeon to approximately $60-65 \%$ more radiation than normal [5]. Secondly, a moderately sized box containing a mirror and camera must be placed in front of the x-ray source, significantly reducing the surgeon's work area.

Here we propose an alternative system to the CamC, the Depth Camera Augmented Fluoroscopy (DeCAF) device, capable of providing real-time visualization of the surgical site by overlaying x-ray images from the C-arm onto live video of the patient's surface anatomy. The $\mathrm{x}$-ray overlay is attained while restricting incursion on the surgeon's work area and allowing the C-arm to be used in its normal position to minimize radiation exposure.

\section{Materials and Methods}

DeCAF uses an Intel RealSense depth camera mounted to the image intensifier tube of a traditional $\mathrm{C}$-arm using a custom 3D printed mount. Using geometric data acquired via the depth camera, DeCAF facilitates transforming a real-time video feed obtained from the side of the C-arm image intensifier aligned with the camera coordinate system to a perspective aligned with the $\mathrm{C}$-arm coordinate system (see Figure 1). The subsequent transformed image of the surface anatomy is then used as an overlay on the acquired x-ray image.

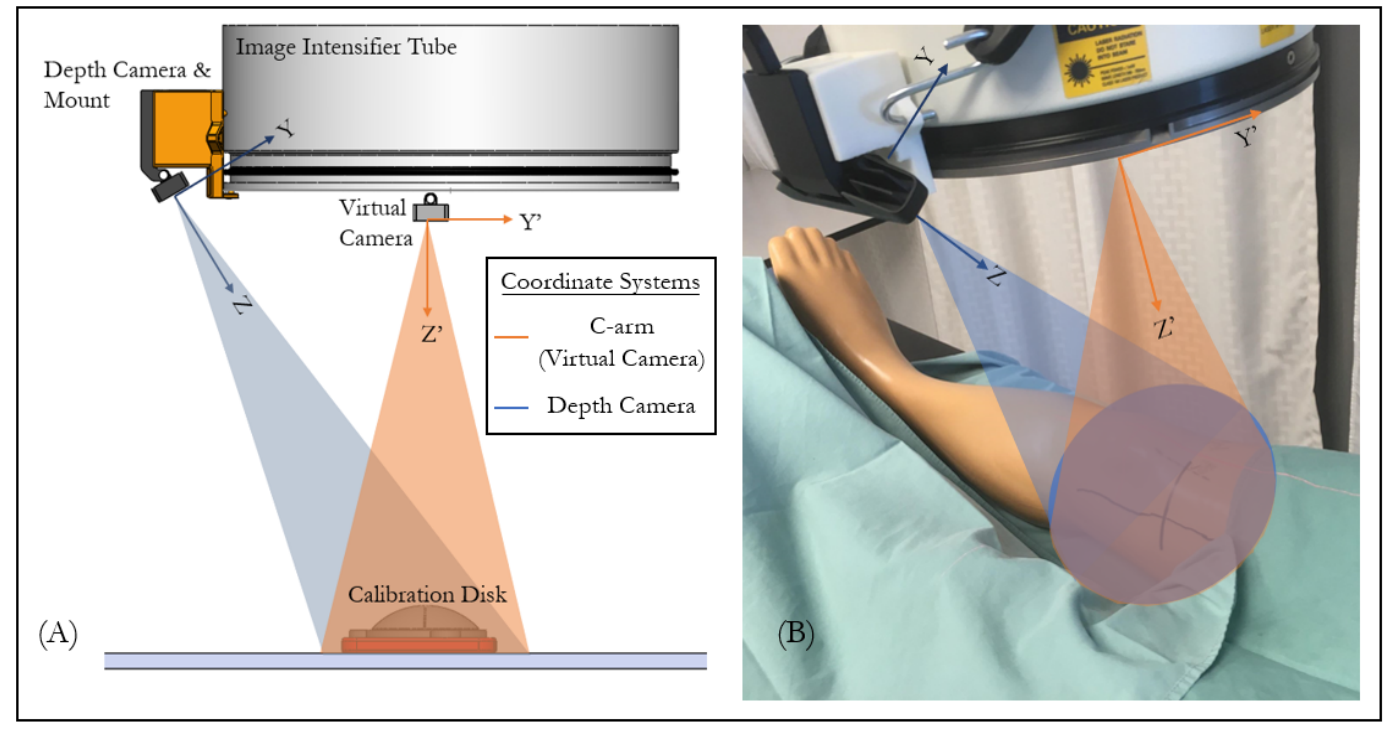

Figure 1: DeCAF C-arm perspectives. (A) Depth camera attached to the image intensifier tube showing actual and virtual coordinate systems. (B) Camera observing a mock surgical scene.

A 3-step calibration procedure is used to determine the coordinate transformation from the perspective of the camera to that of the x-ray source. A calibration disk was developed with four distinct points marked by radio dense ball bearings. The calibration disk is placed in the center of the surgical scene and aligned with the laser targeting system on the C-arm. Using an estimate of the distance from calibration disk to the image intensifier, we establish the transformation from the calibration disk to the 
C-arm ( $\left.\mathrm{T}_{\mathrm{CD}-\mathrm{CA}}\right)$. Geometric data is acquired by the depth camera to determine the transformation from the calibration disk to the depth camera (TCD-DC). The image transformation from the depth camera to the $\mathrm{C}$-arm ( $\left.\mathrm{T}_{\mathrm{DC}-\mathrm{CA}}\right)$ can then be determined.

One challenge with this procedure is that the transformed view is from the image intensifier tube center, meaning that the viewing cone direction from the C-arm Z' axis is opposite to that of the $\mathrm{x}$-ray source. In order to show the x-ray image at the correct size in the overlay, we need to estimate the distance to the relevant anatomic plane where the structures of greatest interest to the surgeon are located. At present, we take an x-ray of the calibration disk showing the four radio-dense markers. The location of these points is used to scale the acquired x-ray images appropriately.

\section{Results}

In a preliminary proof of concept test, DeCAF was tested in a mock surgical setting with an anatomically realistic patient surrogate and used to provide a visual overlay on acquired x-ray images. Figure 2 shows the acquired images and an example of the video overlay feature. Dark areas in the transformed image are areas where video and geometric data are unavailable due to the orientation of the camera relative to the surgical scene.

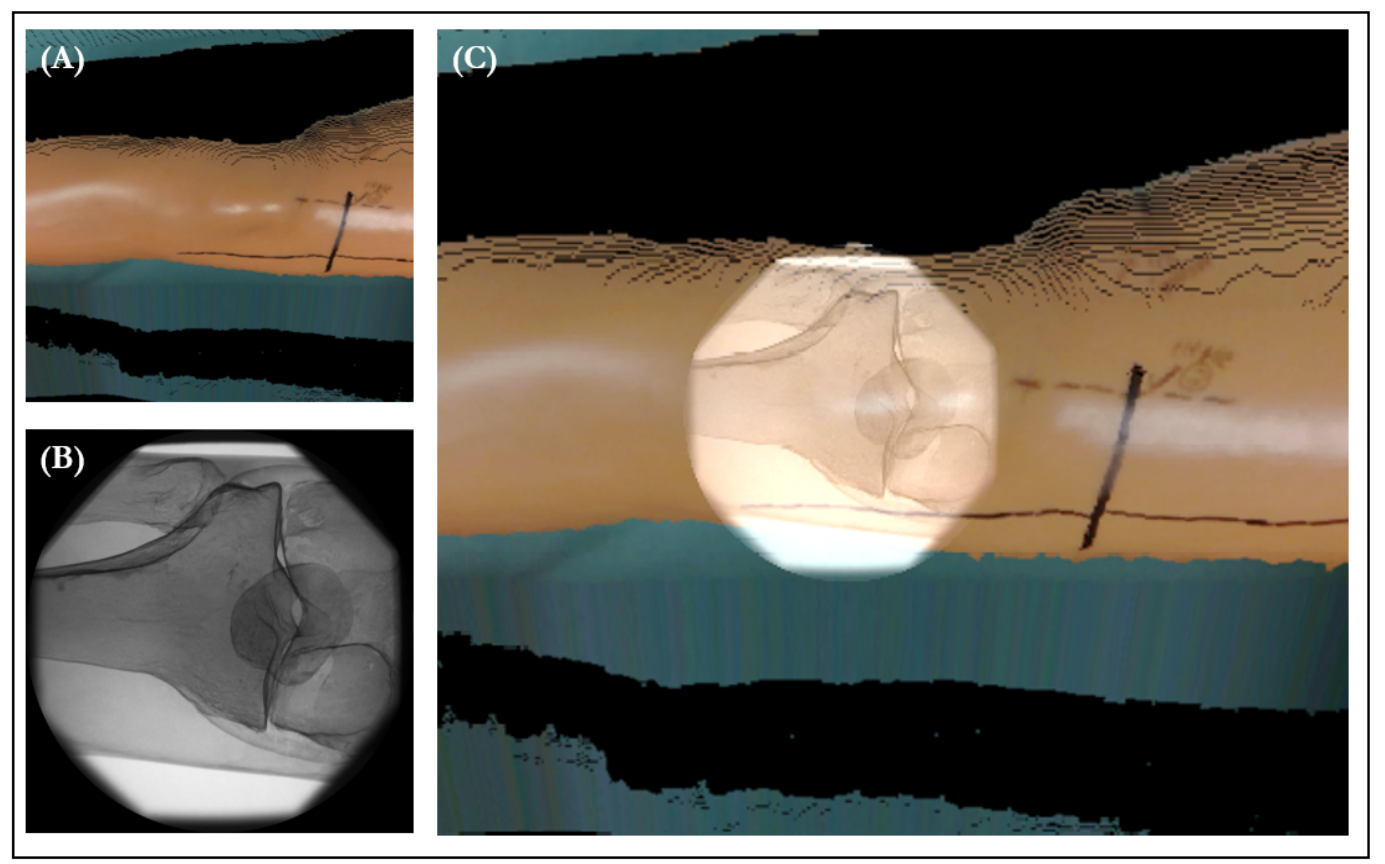

Figure 2: DeCAF video overlay. (A) Transformed image from the depth camera of an anatomically-realistic patient surrogate leg. (B) Acquired x-ray of the knee from C-arm. (C) Overlay image of x-ray and depth camera.

\section{Discussion}

DeCAF successfully replicated the x-ray video overlay feature of the CamC device while eliminating its two key limitations. The depth camera and 3D printed mount measure only $6 \mathrm{~cm} x 7 \mathrm{~cm}$ $\mathrm{x} 12 \mathrm{~cm}$ and is placed to the side of the image intensifier resulting in little or no incursion into the 
surgical space. The device construction allows for the C-arm to be used in the non-inverted position, resulting in significant reduction of radiation exposure to both the patient and attending surgeon compared to the CamC device [3], [5].

Other $\mathrm{C}$-arm augmentation devices exist, one of which was proposed by Reaungamornrat et al. that reconstructs a transformed $\mathrm{x}$-ray image overlay atop a camera video feed achieving submillimeter accuracy [6]. An intraoperative video-based tracking system is mounted on a tripod and placed in the OR to facilitate image transformations. A significant limitation of this approach is the need to use a Carm with spin capabilities to acquire a cone beam $\mathrm{CT}$ image, a radiation intensive and time-consuming step in the OR [6].

Future work will involve evaluating the accuracy by placing radio-dense makers on the surface of an anatomically-realistic patient surrogate. Transformed images will be acquired from the depth camera and overlaid with x-ray images for multiple orientations. Accuracy can be deduced from calculating mean distances from marker positions between the views. If necessary, further software and hardware design iterations will be completed to address any concerns of accuracy and usability. A second depth camera may be added directly across from the first to aid in filling in areas with missing details. Once the design is approved by collaborating surgeons, another design iteration involving bagging of the camera with a sterile cover will be completed to ensure compliance with asepsis requirements prior to evaluating the system in the OR.

\section{References}

[1] T. D. Pinkney and D. G. M. Morton, "Novel approaches to surgical trials and the assessment of new surgical technologies," Br. J. Surg., vol. 102, 2015.

[2] L. Wang, "Novel Techniques for Integrating Video Augmented X-ray Imaging into Orthopedic and Trauma Surgery," Technische Universität München, Fakultät für Informatik, 2012.

[3] N. Navab, S. Heining, and J. Traub, "Camera Augmented Mobile C-Arm (CAMC):," IEEE Trans Med Imaging, vol. 29, no. 7, pp. 1412-1423, 2010.

[4] A. M. von der Heide et al., "Camera-augmented mobile C-arm (CamC): A feasibility study of augmented reality imaging in the operating room, " Int. J. Med. Robot. Comput. Assist. Surg., vol. 14, pp. 1-8, 2018.

[5] M. Tremains, G. Georgiadis, and M. Dennis, "Radiation Exposure W ith Use O $\mathrm{f}$ the InvertedC-Arm Technique in Upper-Extremity Surgery," J. BONE Jt. Surg., 2001.

[6] S. Reaungamornrat, Y. Otake, and A. Uneri, "An on-board surgical tracking and video augmentation system for C-arm image guidance," CARS, pp. 647-665, 2012. 